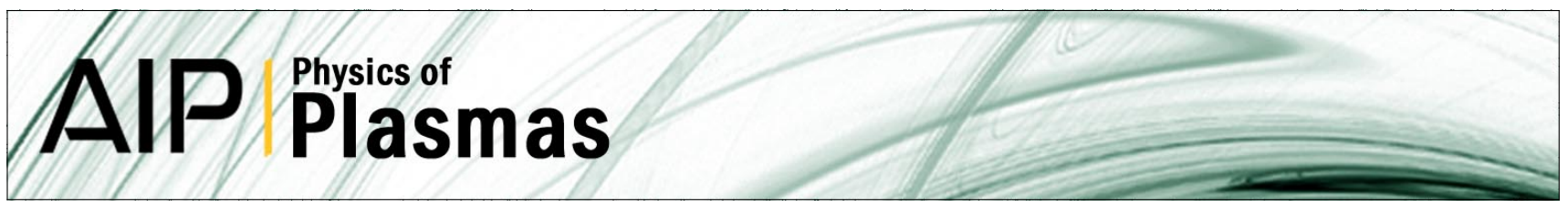

\title{
New role of the lower-hybrid drift instability in the magnetic reconnection
}

Paolo Ricci, J. U. Brackbill, W. Daughton, and Giovanni Lapenta

Citation: Phys. Plasmas 12, 055901 (2005); doi: 10.1063/1.1885002

View online: http://dx.doi.org/10.1063/1.1885002

View Table of Contents: http://pop.aip.org/resource/1/PHPAEN/v12/i5

Published by the American Institute of Physics.

\section{Related Articles}

Inclusion of diamagnetic drift effect in the matching method using finite-width inner region for stability analysis of magnetohydrodynamic modes

Phys. Plasmas 19, 102511 (2012)

On the heterogeneous character of the heartbeat instability in complex (dusty) plasmas

Phys. Plasmas 19, 103701 (2012)

Three-dimensional modeling and analysis of a high energy density Kelvin-Helmholtz experiment

Phys. Plasmas 19, 092112 (2012)

Effect of ion-to-electron mass ratio on the evolution of ion beam driven instability in particle-in-cell simulations Phys. Plasmas 19, 092111 (2012)

Experimental observations of turbulent mixing due to Kelvin-Helmholtz instability on the OMEGA Laser Facility Phys. Plasmas 19, 092702 (2012)

\section{Additional information on Phys. Plasmas}

Journal Homepage: http://pop.aip.org/

Journal Information: http://pop.aip.org/about/about_the_journal

Top downloads: http://pop.aip.org/features/most_downloaded

Information for Authors: http://pop.aip.org/authors

\section{ADVERTISEMENT}

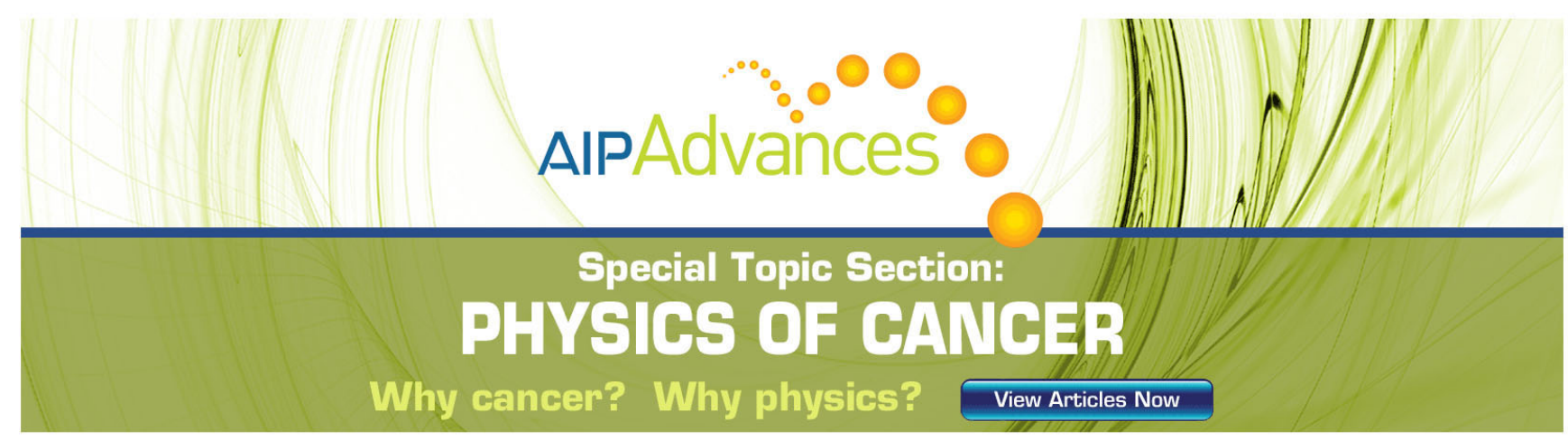




\title{
New role of the lower-hybrid drift instability in the magnetic reconnection ${ }^{a)}$
}

\author{
Paolo Riccib) \\ Department of Physics and Astronomy, Dartmouth College, Hanover, New Hampshire 03755 \\ J. U. Brackbill ${ }^{\mathrm{c})}$ \\ ParticleSolutions, Portland, Oregon 97214 \\ W. Daughton ${ }^{\text {d) }}$ \\ University of Iowa, Iowa City, Iowa 52242 \\ Giovanni Lapenta ${ }^{\mathrm{e})}$ \\ Istituto Nazionale per la Fisica della Materia (INFM), Unità del Politecnico di Torino, \\ Corso Duca degli Abruzzi 24-10129 Torino, Italy and Los Alamos National Laboratory, \\ Los Alamos, New Mexico 87545
}

(Received 22 November 2004; accepted 7 February 2005; published online 22 April 2005)

\begin{abstract}
Kinetic simulation results reveal that the growth of the lower-hybrid drift instability (LHDI) in current sheets has an important effect on the onset and nonlinear development of magnetic reconnection. The LHDI does this by heating electrons anisotropically, by increasing the peak current density, by producing current bifurcation, and by causing ion velocity shear. The role of these in magnetic reconnection is explained. Confidence in the results is strongly enhanced by agreement between implicit and massively-parallel-explicit particle-in-cell simulations. (C) 2005 American Institute of Physics. [DOI: 10.1063/1.1885002]
\end{abstract}

\section{INTRODUCTION}

The importance of the lower-hybrid drift instability (LHDI) in the evolution of current sheets with thickness comparable to the thermal ion gyroradius has been studied for decades. The topic is of central relevance to the physics of magnetic reconnection and has widespread application to space, astrophysical, and laboratory plasmas. ${ }^{1}$ Early work on LHDI by Krall and Liewer ${ }^{2}$ has been followed by numerous papers (see Ref. 1 for a review of the literature).

The LHDI is driven by the diamagnetic current that arises because of the presence of inhomogeneities in the density and magnetic field in a current sheet. The fastest growing modes are primarily electrostatic, and are confined to the edge region with $k \rho_{e} \sim 1, \omega \sim k U_{i} \leqslant \Omega_{\mathrm{lh}}, \gamma \leqslant \Omega_{\mathrm{lh}}$, where $\rho_{e}$ is the electron gyroradius, $U_{i}$ the ion diamagnetic drift velocity, $\Omega_{\mathrm{lh}} \approx \sqrt{\Omega_{c e} \Omega_{c i}}$ is the lower-hybrid drift frequency $\left(\Omega_{c e}\right.$ and $\Omega_{c i}$ are the electron and the ion gyrofrequencies, respectively). ${ }^{3}$ Longer wavelength LHDI modes, $k \sqrt{\rho_{i} \rho_{e}} \sim 1$, show a slower growth rate, $\gamma \sim \Omega_{c i}$, and have a significant electromagnetic component that can penetrate into the central region for sufficiently thin current layers. ${ }^{4}$

Since it is a relatively short wavelength and high frequency instability, the LHDI has been considered for some years a source of anomalous resistivity, ${ }^{5,6}$ and it is often invoked to explain the discrepancy between the rapid observed rate of reconnection and the slow, theoretically predicted Sweet-Parker rate based on classical resistivity. Indeed the

\footnotetext{
Paper PI1B 2, Bull. Am. Phys. Soc. 49, 286 (2004).

${ }^{b)}$ Invited speaker. Electronic mail: paolo.ricci@dartmouth.edu

${ }^{c)}$ Electronic mail: jerrybrackbill@ comcast.net

d)Electronic mail: william-daughton@uiowa.edu

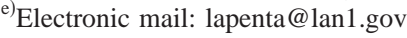

LHDI has been observed during magnetic reconnection in the magnetotail, ${ }^{7}$ in the magnetopause, ${ }^{8}$ and in laboratory experiments. ${ }^{9}$ However, two problems make the explanation of anomalous resistivity based on LHDI difficult to accept. First, both simulations ${ }^{10}$ and observations ${ }^{7,9}$ show that the saturation level of the LHDI is determined primarily by the electron dynamics, and it is far too low to justify the anomalous resistivity that is required to obtain realistic reconnection rates within the Sweet-Parker model. Second, the LHDI is mostly stabilized at high plasma $\beta$ and thus it grows primarily on the flanks of the current sheet, ${ }^{11}$ unless the current layer is very thin. ${ }^{4}$

Recently, the effects of the LHDI have been revisited. ${ }^{12-18}$ In the present paper, it is shown that the LHDI may play an important role in the onset of magnetic reconnection, but through a mechanism very different in nature than anomalous resistivity. Although the LHDI is a microscopic instability, it is also responsible for macroscopic changes in the current sheet. The LHDI heats electrons anisotropically, preferentially in the perpendicular direction, peaks and bifurcates the current sheet, and creates ion velocity shear. ${ }^{14,15}$ These effects have important consequences both on reconnection onset and on the saturation of reconnection. In particular, simulation results show that both temperature anisotropy with $T_{e \perp} / T_{e \|}>1$ and current peaking strongly enhance the linear growth rate of the tearing instability. ${ }^{14}$

We note that the role of electron anisotropy on the onset of other electron anisotropy driven instabilities was recently reexamined for a neutral sheet. ${ }^{19}$ It has been previously argued that any electron anisotropy would be isotropized on a very fast time scale due to a variety of electron anisotropy driven modes, such as the whistler anisotropy instability. 
However, it does not appear that these modes really exist within a neutral sheet. The new results indicate that for $T_{\perp e} / T_{\| e}>1$ the anisotropic tearing mode is the dominant instability while in the opposite limit $T_{\perp e} / T_{\| e}<1$ the current aligned Weibel instability is driven unstable. ${ }^{19}$ Thus the electron anisotropy may in fact play a crucial role in governing the onset physics. ${ }^{19}$

This paper reviews published results and introduces some new results on the scaling of LHDI evolution with the current sheet thickness. The scaling enables us to show that the effect of the LHDI decreases with the thickness of the current sheet profile, passing from current peaking in the central region to current bifurcation. Moreover, it is shown that the level of the electron anisotropy induced by the LHDI is reduced for thicker current sheets.

The paper is organized as follows. Section II describes the system we study and the simulation tools we use for the task. Section III focuses on the macroscopic effects of the LHDI, which are explained by a simple physical model in Sec. IV. Section V reviews results on the effect of the LHDI on the linear and nonlinear evolution of the tearing instability.

\section{PHYSICAL SYSTEM AND THE SIMULATION APPROACH}

Following the Geospace Environment Modeling (GEM) magnetic reconnection challenge, ${ }^{20}$ a Harris current sheet is considered. ${ }^{21}$ The usual magnetotail reference frame is used, with the magnetic field aligned to the $x$ direction,

$$
\mathbf{B}=B_{0} \tanh (z / L) \mathbf{e}_{x},
$$

and plasma density given by

$$
n=n_{0} \operatorname{sech}^{2}(z / L) \text {. }
$$

The current sheet parameters are $\omega_{p e} / \Omega_{c e}=2.88, T_{i} / T_{e}$ $=5, m_{i} / m_{e}=180$, and three different current sheet thicknesses are considered, $\rho_{i} / L=1.828,0.915$, and $0.457 \quad\left(v_{\text {th }, s}\right.$ $\left.=\sqrt{2 T_{s} / m_{s}}\right)$. The boundary conditions are periodic for both particles and fields in the $x$ direction (for simulation in the tearing plane) and $y$ direction (for simulations in the LHDI plane). Conducting boundaries are imposed for the fields at the $z$ boundaries, and reflecting boundary conditions are used for the particles. The domain considered is $L_{y} \times L_{z}=12 L$ $\times 12 L$.

To investigate the evolution of the system, a linear Vlasov code and two different nonlinear particle-in-cell (PIC) simulation codes are used. The linear Vlasov results use the formally exact approach described in Refs. 4 and 22. This technique employs a normal mode calculation using a full Vlasov description for both ions and electrons. The orbit integrals arising from the linear Vlasov theory are treated numerically using the exact unperturbed particle orbits and including the form of the perturbation inside the integral. Both electromagnetic and electrostatic contributions to the field perturbation are retained and resulting system of integrodifferential equations is solved using a finite element expansion of the eigenfunction. ${ }^{4}$
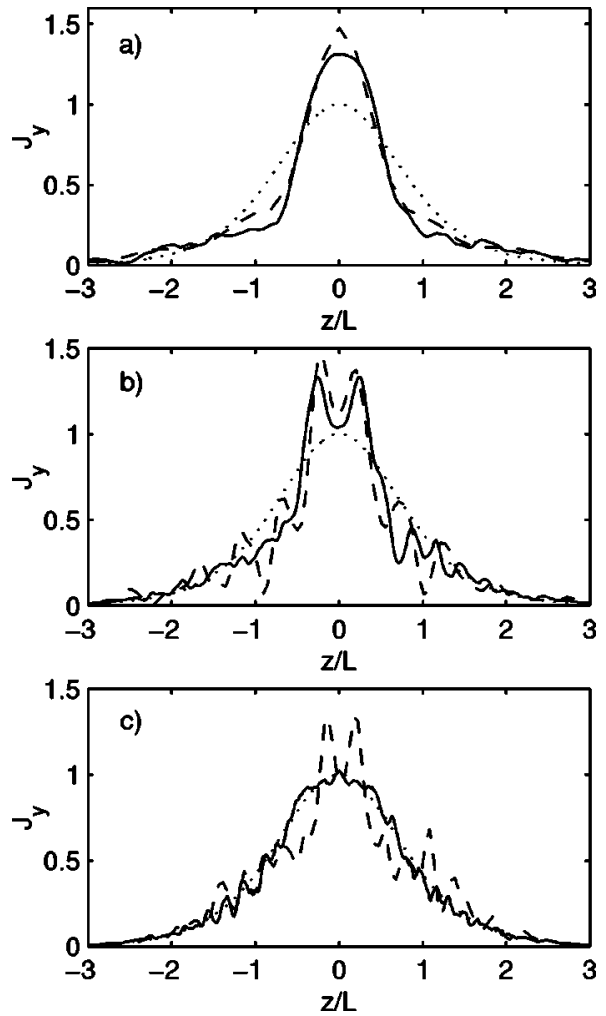

FIG. 1. The evolution of the current density $J_{y}$ is shown from simulations in the $(y, z)$ plane. The dotted lines represent the current profiles at $t=0$ averaged along $y$. Profiles at $t \Omega_{c i}=4$ are shown by a solid line (NPIC simulation) and a dashed line (CELESTE3D simulation) for $\rho_{i} / L=1.828$, case (a); at $t \Omega_{c i}=8$ for the $\rho_{i} / L=0.915$, case (b); and at $t \Omega_{c i}=17$ for the $\rho_{i} / L=0.457$, case (c). The other current sheet parameters are $\omega_{p e} / \Omega_{c e}=2.88, m_{i} / m_{e}$ $=180$, and $T_{i} / T_{e}=5$.

The nonlinear dynamics are simulated by two PIC codes, an explicit simulation code NPIC and an implicit simulation code CELESTE3D. The explicit plasma simulation code NPIC is based on a well-known explicit electromagnetic algorithm. $^{23,24}$ The particle trajectories within NPIC are advanced using the leapfrog technique, and particle moments are accumulated with area weighting. The simulations are run on a massively parallel computer. The implicit plasma simulation code CELESTE3D (see Refs. 25-27) solves the full set of Maxwell-Vlasov equations using the implicit moment method. Both Maxwell's and Newton's equations are discretized implicitly in time. The implicit simulations are run on a workstation.

The nonlinear simulations are performed by the two codes with very different simulation parameters, as discussed in Ref. 14.

\section{NONLINEAR EVOLUTION OF THE LHDI}

Two-dimensional simulations are performed in the $(y, z)$ plane of the Harris current sheet with several current sheet thicknesses. The initial Harris equilibrium is unstable to the LHDI and the early dynamics is dominated by this instability. In the present paper the focus is on the nonlinear consequences of the LHDI.

The LHDI modifies the current profile. In Fig. 1 the $y$-averaged current profile for the three current sheets thick- 
nesses is represented after the growth of the LHDI. For the thinnest case, $\rho_{i} / L=1.828$, the LHDI causes a peaking of the current profile. This effect has been documented in previous works ${ }^{12-14,16-18,28}$ and the alteration is primary due to electron acceleration: the ion and electron density modifications are weaker. ${ }^{12,13,16}$ Within a fluid model, current peaking due to LHDI was also predicted in Ref. 29. At a later stage in the evolution of the current sheet, a strong kink instability dominates the evolution of the current sheet. ${ }^{16-18}$ Simulations have revealed that the current profile becomes bifurcated at this stage. ${ }^{30}$

In the simulation of the current sheet with intermediate thickness, both NPIC and CELESTE3D show bifurcation of the current. Current bifurcation has been observed in satellite measurements of thin $\left(L \sim \rho_{i}\right)$ (see Ref. 31) and thick current sheets $\left(L>\rho_{i}\right)$ (see Refs. 32 and 33), and in laboratory experiments. ${ }^{34}$ The build-up of the bifurcated current sheet as a result of the LHDI was also recently shown in particle simulations by Sitnov et al., ${ }^{35}$ starting from a non-Harris current sheet equilibrium. It should be remarked that current sheet bifurcation has also been explained as a consequence of the kink that affects the dynamics of the current sheets ${ }^{36}$ or as a consequence of the physics in the reconnection plane. . $^{37,38}$

For the thickest current sheet, the agreement between NPIC and CELESTE3D is poor. We note that this simulation is challenging for both codes and it is our plan to understand the reason of the discrepancies between the two simulation results. While CELESTE3D shows current bifurcation, NPIC reveals small fluctuations of the current on the flanks of the current sheet. Nevertheless, it should be pointed out that both NPIC and CELESTE3D reveal no significant changes of the current density at $z=0$, and that the peak value of the bifurcated current shown by CELESTE3D is smaller than the one observed in the thinner current sheet. Thus, both codes show that the effect of the LHDI decreases with the current sheet thickness.

The nonlinear evolution of the LHDI not only heats electrons (an effect documented in many previous works, e.g., Ref. 9) but preferentially heats electrons in directions perpendicular to the magnetic field. The ratio of perpendicular to parallel electron temperature, evaluated as the ratio of the components of the electron pressure tensor, $T_{e \perp} / T_{e \| l}$, from NPIC is shown in Fig. 2. The electron distribution functions are not gyrotropic in the thinnest current sheet case. Perpendicular heating decreases with increases in the current sheet thickness. The values of anisotropy at the center of the current sheet from NPIC and CELESTE3D are listed in Table I.

Finally, it is interesting to note that the LHDI creates velocity shear. This latter consequence has been the subject of a number of papers (see Ref. 16 and references therein). Its main effect is to promote the growth of a KelvinHelmholtz instability (KHI). Recent three-dimensional simulations show that the KHI appears not to have an important effect on reconnection onset since it grows on the same reconnection time scales, ${ }^{14}$ but its role on reconnection onset in more general configuration is still to be investigated. ${ }^{16}$
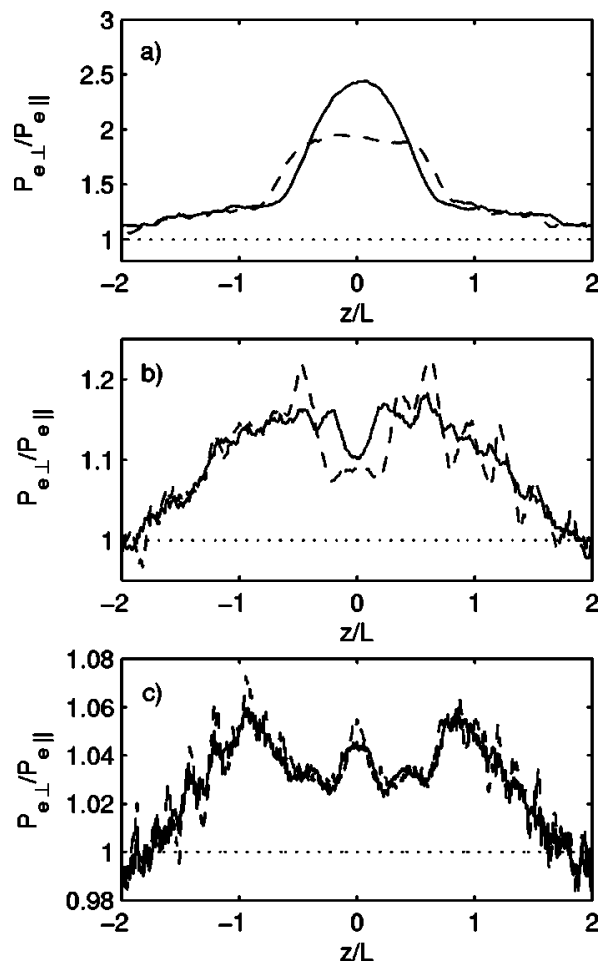

FIG. 2. The electron pressure ratios $P_{e z z} / P_{e x x}$ (solid line) and $P_{e y y} / P_{e x x}$ (dashed line) are averages along $y$ and plotted at time $t \Omega_{c i}=4$ for the $\rho_{i} / L$ $=1.828$, case (a); at $t \Omega_{c i}=8$ for the $\rho_{i} / L=0.915$, case (b); and at $t \Omega_{c i}=17$ for the $\rho_{i} / L=0.457$, case (c). The other current sheet parameters are $\omega_{p e} / \Omega_{c e}$ $=2.88, m_{i} / m_{e}=180$, and $T_{i} / T_{e}=5$. The results are from the NPIC simulations.

\section{A SIMPLE PHYSICAL EXPLANATION}

A simple physical model is sufficient to explain the consequences of the LHDI. We refer the reader to Refs. 15 and 17 for a complete description of the process, and in the following we summarize the basic mechanism. Particles can be distinguished between crossing, with trajectories that traverse both sides of the current layer, and noncrossing, with trajectories that are confined to one side of the layer. The perpendicular kinetic energy, $\epsilon=m_{s}\left(v_{z}^{2}+v_{y}^{2}\right) / 2$, and canonical momentum, $p_{y}=m_{s} v_{y}+q_{s} A_{y} / c \quad\left(A_{y}\right.$ $=-B_{0} L \ln [\cosh (z, L)]$ in a Harris sheet), are constants of the particle motion, and only particles with $\epsilon>p_{y}^{2} / 2 m_{s}$ can cross the layer. The boundary between crossing and noncrossing particles can also be written as

$$
\frac{v_{y}}{v_{\mathrm{th}, s}}=\frac{\alpha}{2}-\frac{1}{2 \alpha}\left(\frac{v_{z}}{v_{\mathrm{th}, s}}\right)^{2},
$$

where $\alpha=\left(L / \rho_{s}\right) \ln [\cosh (z / L)]$. The approximate phase velocity of the LHDI for cold electrons is $\omega / k_{y} \approx U_{i} / 2$ and it is

TABLE I. Electron pressure anisotropy $P_{e \perp} / P_{e \|}$ at the center of the current sheet for different thicknesses of the current sheet at time $t \Omega_{c i}=4\left(\rho_{i} / L\right.$ $=1.828), t \Omega_{c i}=8\left(\rho_{i} / L=0.915\right)$, and $t \Omega_{c i}=17\left(\rho_{i} / L=0.457\right)$.

\begin{tabular}{lccc}
\hline \hline & $\rho_{i} / L=1.828$ & $\rho_{i} / L=0.915$ & $\rho_{i} / L=0.457$ \\
\hline NPIC & 2.20 & 1.09 & 1.04 \\
CELESTE3D & 1.84 & 1.01 & 0.95 \\
\hline \hline
\end{tabular}




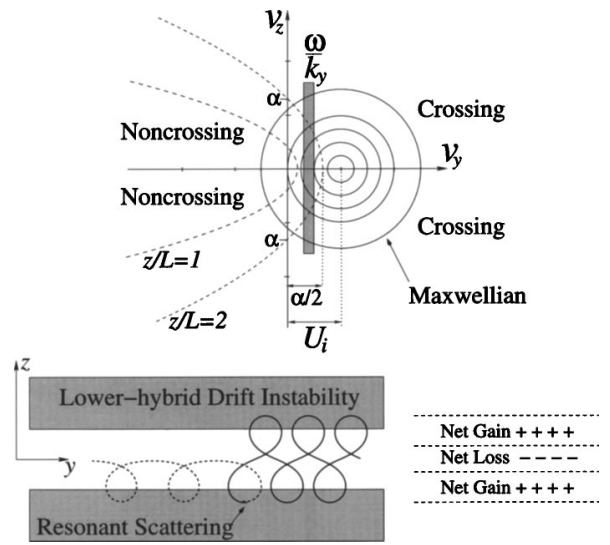

FIG. 3. Cross section of phase space (top) in the $v_{z}-v_{y}$ plane, illustrating a drifting Maxwellian ion distribution and the phase space boundary in Eq. (3) for two different spatial positions in the layer. The shaded region in the upper figure corresponds to the approximate phase velocity of the waves $\omega / k_{y} \approx U_{i} / 2$, which are in the proper region to resonantly scatter crossing ions into the noncrossing region of phase space. This scattering process and the resulting charge accumulation is illustrated in the lower figure.

in the proper region to permit resonant scattering of ions from crossing trajectories to noncrossing trajectories and vice versa. As is shown in Fig. 3, because of the slope of the distribution function in the vicinity of the resonance, the scattering from the crossing to the noncrossing region is more frequent. It should be remarked that scattering can only occur if the ion meandering length $\sqrt{2 \rho_{i} L}$, which measures the spatial extent of the crossing ion orbits, overlaps with the localization of the LHDI.

Scattering has two consequences: (i) it leads to a loss of positive charge in the center, in conjunction with a gain in the edge region, and therefore gives rise to an electrostatic potential structure across the layer; (ii) since the ion scattering is asymmetric in $v_{y}$ (particles with smaller $v_{y}$ are preferentially scattered), a significant velocity shear is induced at the edge of the current sheets ${ }^{17}$ that induce a KHI. ${ }^{18}$

The electron flow velocity can be approximated as

$$
V_{e y} \approx U_{e}-\frac{c}{B_{x}} \frac{\partial \phi}{\partial z}
$$

where the first term is the equilibrium flow velocity, the second term is the $\mathbf{E} \times \mathbf{B}$ velocity induced by the electrostatic potential, the inertia terms are neglected, and the equilibrium distributions are used to evaluate the pressure tensor. As is shown in Fig. 4, the agreement between the velocity evaluated from Eq. (4) and the actual velocity resulting from NPIC simulation is remarkable at early stage of the current sheet evolution, before the current sheet profile strongly deviates from its initial shape.

Electron anisotropy is closely related to the plasma sheet structure. Anisotropic heating can be explained considering that for the case of noncrossing electrons with helical trajectories, the magnetic moment $\mu=m v_{\perp}^{2} /\left(2 B_{x}\right)$ is an adiabatic invariant, since the time scales of the evolution of the system are slow compared to the typical electron scales. This implies that the perpendicular temperature is related to the local magnetic field,
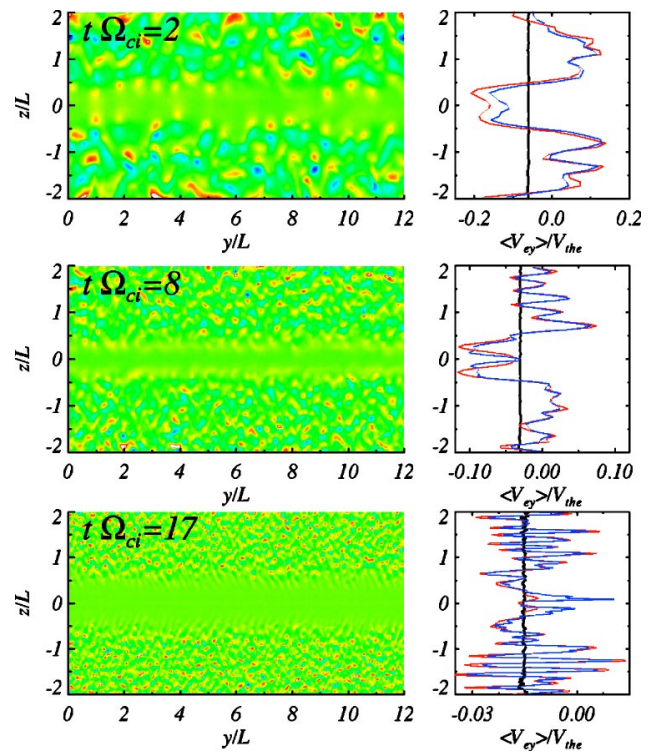

FIG. 4. (Color online). The $y$-averaged, $y$-velocity profile is shown from the NPIC simulation (red line) and compared with the estimate of Eq. (4) (blue line). The three cases are $\rho_{i} / L=1.828$ (top), $\rho_{i} / L=0.915$ (middle), and $\rho_{i} / L=0.457$ (bottom). The black line is the initial velocity profile at $t=0$.

$$
\frac{T_{e \perp}(z, t)}{T_{e \perp}(z, t=0)} \approx \frac{B_{x}(z, t)}{B_{x}(z, t=0)} .
$$

Where the electron orbits are helical, this expression provides a good estimate of the perpendicular heating at early stage of the current sheet evolution, as shown in Fig. 5. In the central region, the electron trajectories undergo a variety of complicated crossing orbits and $\mu$ is no longer the relevant adiabatic invariant. [The comparison for the thickest case is poor, more likely because the actual physical heating due to Eq. (5) is masked by a fair amount of numerical heating, rather than because other physical mechanisms produce electron anisotropic heating.]
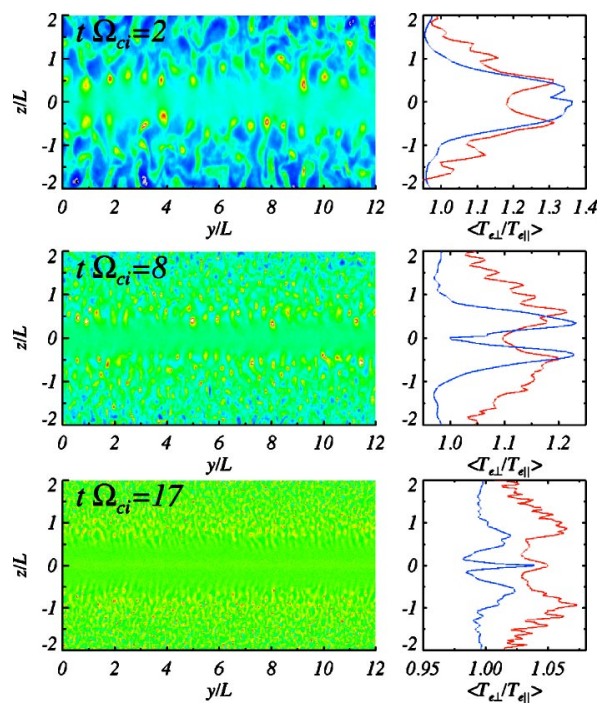

FIG. 5. (Color online). The $y$-averaged $T_{e \perp} / T_{e \|}$ profile is shown from the NPIC simulation (red line) and compared with the estimate of Eq. (5) (blue line). The three cases are $\rho_{i} / L=1.828$ (top), $\rho_{i} / L=0.915$ (middle), and $\rho_{i} / L=0.457$ (bottom). 


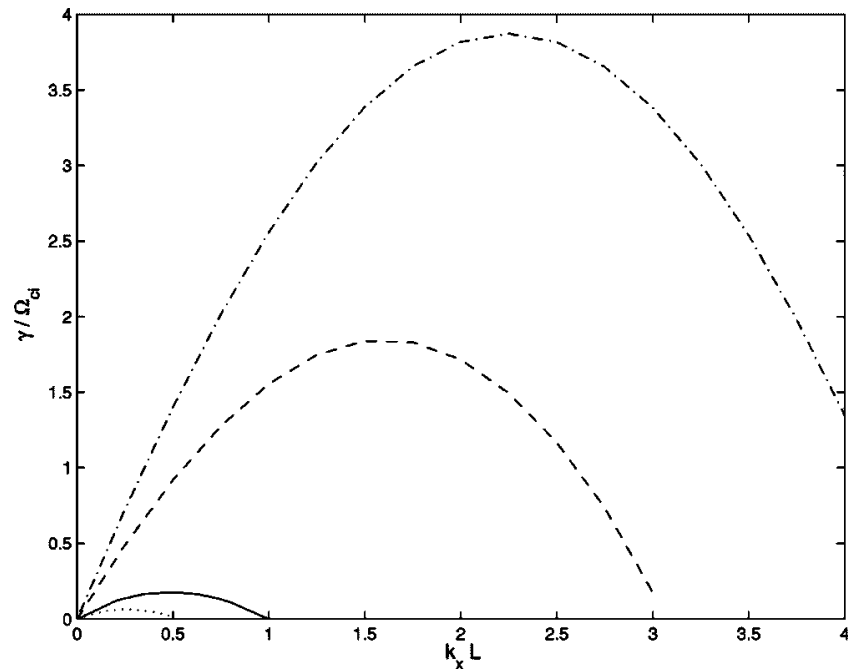

FIG. 6. The growth rate $\gamma / \Omega_{c i}$ of the tearing mode is plotted as a function of $k_{x} L$ for $T_{e \perp} / T_{e \|}=0.9$ (dotted), $T_{e \perp} / T_{e \|}=1$ (solid), $T_{e \perp} / T_{e \|}=1.5$ (dashed), $T_{e \perp} / T_{e \|}=2$ (dash dotted). The other current sheet parameters are $\omega_{p e} / \Omega_{c e}$ $=2.88, m_{i} / m_{e}=180, \rho_{i} / L=1.828$, and $T_{i} / T_{e \|}=5$.

\section{EFFECT OF LHDI ON THE TEARING INSTABILITY}

We focus attention on the effect of the nonlinear consequences of the LHDI on the tearing mode in the case $\rho_{i} / L$ $=1.828$. In two-dimensional simulations without an initial perturbation, linear analysis and simulations give the same growth rate for the fastest growing tearing mode, $\gamma$ $=0.176 \Omega_{c i}$, but tearing saturates at such a low amplitude that only a thin reconnected region results. The half-width, $w$ $\approx 0.46 L$ at $t \Omega_{c i}=83$, should be compared with the GEM challenge with a large initial perturbation, which covers the whole domain, $w \approx 10 L$ at $t \Omega_{c i} \approx 30 .^{14}$

Nonlinear theories (e.g., see Refs. 39-41) predict that anisotropic heating of electrons during the growth of the instability reduces $T_{e \perp} / T_{e \|}$ below 1 , which strongly stabilizes tearing. Two-dimensional simulations performed by NPIC and CELESTE3D confirm the prediction and show that by $t \Omega_{c i}=80, T_{e \perp} / T_{e \|}$ is reduced from 1 to the range $0.83-0.87$, for which the linear code predicts that the maximum growth rate, $\gamma=0.04 \Omega_{c i}$, is a fraction of its initial value. ${ }^{14}$ For a larger system, small islands will eventually coalesce into larger islands, but this process is too slow to account for the rapid reconnection observed in simulations extended in the third dimension. ${ }^{14}$

The LHDI also heats electrons anisotropically, but in contrast to the tearing instability, it increases rather than decreases $T_{e \perp} / T_{e \|}$. The linear growth rates of the tearing instability obtained by the linear Vlasov code, Fig. 6, increases with $T_{e \perp} / T_{e \|}$ : for $T_{e \perp} / T_{e \|}=2$ the maximum growth rate, $\gamma$ $\approx 3.8 \Omega_{c i}$, is more than an order of magnitude larger than the maximum growth rate, $\gamma \approx 0.18 \Omega_{c i}$, for $T_{e \perp} / T_{e \|}=1$. The wavelength of the fastest growing tearing mode decreases with increasing anisotropy: in the case $T_{e \perp} / T_{e \|}=2$ the maximum occurs for $k_{x} L=2.25$, considerably larger than the typical wavelength, $k_{x} L=0.5$, for isotropic electrons. These results are consistent with earlier analysis. ${ }^{42-47}$

Since the anisotropic heating due to the LHDI occurs more rapidly than tearing growth, we consider simulations a)
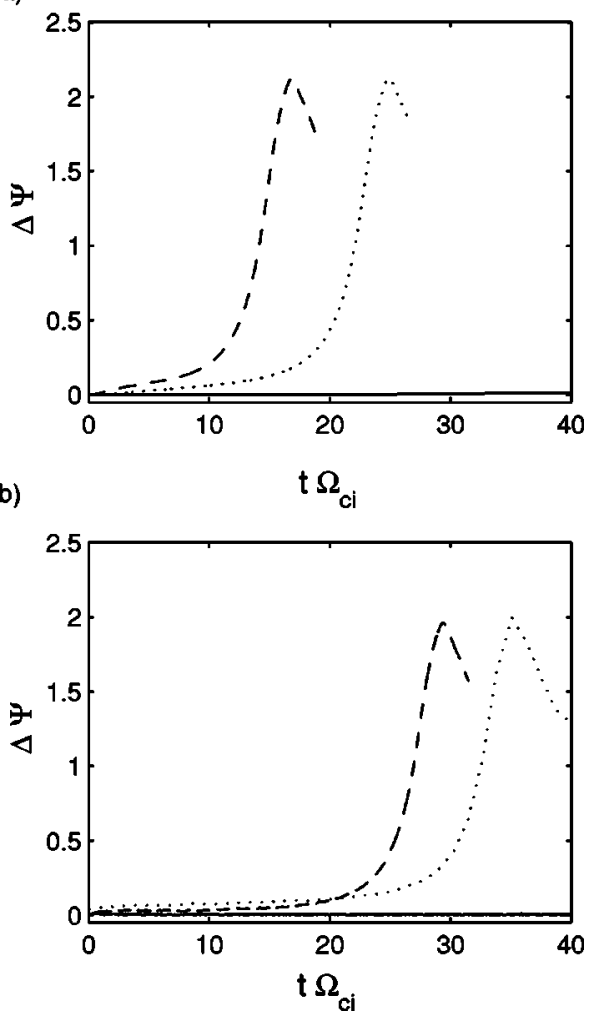

FIG. 7. The change in the reconnected flux $\Delta \Psi$ with time is compared for NPIC (a) and CELESTE3D (b) simulations. The parameters of the current sheets are $T_{e \perp} / T_{e \|}=1$ (solid), $T_{e \perp} / T_{e \|}=1.5$ (dotted), $T_{e \perp} / T_{e \|}=2$ (dashed), $\omega_{p e} / \Omega_{c e}=2.88, m_{i} / m_{e}=180, \rho_{i} / L=1.828$, and $T_{i} / T_{e \|}=5$.

with an anisotropic electron temperature as an initial condition. With $T_{e \perp} / T_{e \|}>1$, NPIC and CELESTE3D simulations show that short wavelength modes grow to form small islands, which then merge to form a single large island (the dominant mode number $m_{x}=1$ covers the whole domain by $\left.t \Omega_{c i} \approx 20\right)$. The reconnected flux is shown in Fig. 7. For $T_{e \perp} / T_{e \|}$ between 1.5 and 2 , reconnection involves the whole domain and the growth of the tearing instability does not saturate until all of the available magnetic flux is reconnected, similar to the GEM challenge. Consistent with earlier results, the ratio $T_{e \perp} / T_{e \|}$ decreases to $\approx 0.8$ due to tearing, but then increases with the onset of fast reconnection. ${ }^{14}$

Our results and others (see Refs. 12 and 13) show that thinner current sheets and higher current densities also enhance the linear growth rate and nonlinear evolution of the tearing instability. The linear growth rate, Fig. 8, is higher for thinner current sheets: $\gamma=0.176 \Omega_{c i}$ for $\rho_{i} / L=1.828$ (GEM challenge thickness) compared with $\gamma=0.632 \Omega_{c i}$ for $\rho_{i} / L$ $=3.656$. The wavelength of the fastest growing mode, however, is the same in all cases. For sufficiently thin current sheets, the tearing mode does not saturate at low levels and covers the whole domain. The reconnected flux increases until it exhausts the supply by $t \Omega_{c i} \approx 20{ }^{14}$

\section{CONCLUSIONS}

The evolution of the LHDI and its influence on reconnection is studied using results from a linear kinetic code and two PIC codes employing very different algorithms: NPIC is 


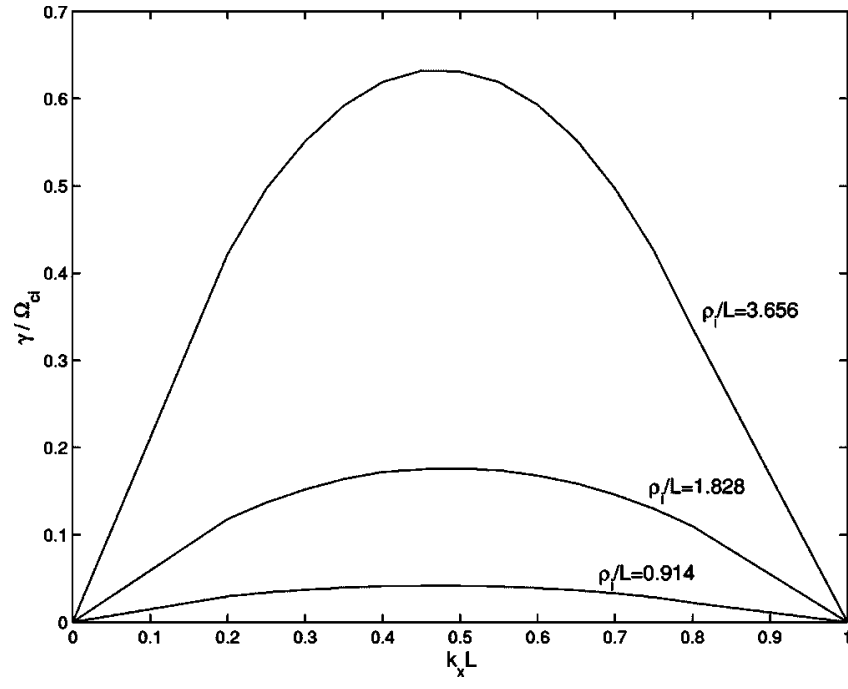

FIG. 8. The growth rate $\gamma / \Omega_{c i}$ of the tearing mode as a function of $k_{x} L$ is plotted for the plasma parameters $\omega_{p e} / \Omega_{c e}=2.88, m_{i} / m_{e}=180, T_{i} / T_{e}=5$, and different values of $\rho_{i} / L$.

a massively parallel explicit code and CELESTE3D is a implicit-moment method PIC code.

LHDI produces strong changes in the current profile. Depending on the current sheet thickness, current peaking or current bifurcation are observed. The changes in the current profile decrease with the thickness of the current sheet. Moreover, LHDI causes anisotropic heating of electrons. Also anisotropic heating decreases with the thickness of the current sheet.

The effects of the LHDI can be explained with a simple physical model. The nonlinear evolution of the LHDI gives rise to a resonant scattering of ions into the noncrossing region of space. This in turn produces an electrostatic potential structure across the sheet leading to a strong change of the current density profile and perpendicular electron heating through an adiabatic process.

It is shown that both current peaking and anisotropic heating of the current sheet are effective in enhancing the linear growth rate of tearing instability. Although it is clear that the peaking of a monotonic current profile will enhance the growth rate of collisionless tearing, the influence of a bifurcated current profile on the stability of the tearing mode deserves a careful investigation that recent simulations performed with CELESTE3D have started to approach. ${ }^{48}$ With a favorable anisotropy and a thin enough current sheet, the tearing instability grows large enough to decouple electrons and ions and reconnection can encompass the whole domain. The scaling study indicates that the effect of the LHDI is reduced with the thickness of the current sheet: when the spatial extent of the crossing ion orbits intersect with the region of strong LHDI activity, the onset mechanism is triggered leading to a bifurcated current profile and anisotropic heating.

It should be remarked that the approach followed in the present study considers an isotropic and single peaked equilibrium, like the Harris current sheet, that is affected by the LHDI. The LHDI modifies the current profile and makes the electron distribution anisotropic: the same relation between current sheet profiles and anisotropic electron distribution has been pointed out in the study of current sheet equilibria. Within the Grad-Shafranov theory ${ }^{49}$ and the Vlasov theory, ${ }^{35,50}$ it has been shown that an anisotropic electron distribution $\left(T_{e \perp}>T_{e \|}\right)$ leads to a bifurcated current profile.

Finally, it is important to note that the initial configuration examined in this study did not include a background plasma (lobe) nor did it examine the influence of a guide field or normal component. Each of these factors is known to influence the stability properties of the LHDI, so further work is needed to examine the feasibility of this onset mechanism for more realistic conditions.

\section{ACKNOWLEDGMENTS}

The authors gratefully acknowledge useful discussions with B. Coppi, I. Furno, J. Huba, and B. Rogers.

This research was supported by the LDRD program at the Los Alamos National Laboratory by the U.S. Department of Energy, under Contract No. W-7405-ENG-36, and by NASA, under the "Sun Earth Connection Theory Program" and the "Geospace Sciences Program."

${ }^{1}$ D. Biskamp, Magnetic Reconnection in Plasmas (Cambridge University Press, Cambridge, 2000).

${ }^{2}$ N. A. Krall and P. C. Liewer, Phys. Rev. A 4, 2094 (1971).

${ }^{3}$ R. C. Davidson, N. T. Gladd, and C. Wu, Phys. Fluids 20, 301 (1977).

${ }^{4}$ W. Daughton, Phys. Plasmas 10, 3103 (2003).

${ }^{5}$ R. C. Davidson and N. T. Gladd, Phys. Fluids 18, 1327 (1975).

${ }^{6}$ J. D. Huba, N. T. Gladd, and K. Papadopoulos, Geophys. Res. Lett. 4, 125 (1977).

${ }^{7}$ I. Shinohara, T. Nagai, M. Fujimoto et al., J. Geophys. Res., [Space Phys.] 103, 20365 (1998).

${ }^{8}$ S. Bale, F. Mozer, and T. Phan, Geophys. Res. Lett. 29, 2180 (2002).

${ }^{9}$ T. A. Carter, H. Ji, F. Trintchouk, M. Yamada, and R. M. Kulsrud, Phys. Rev. Lett. 88, 015001 (2002).

${ }^{10}$ J. U. Brackbill, D. W. Forslund, K. B. Quest, and D. Winske, Phys. Fluids 27, 2682 (1984).

${ }^{11}$ J. D. Huba, J. F. Drake, and N. T. Gladd, Phys. Fluids 23, 552 (1980).

${ }^{12}$ M. Sholer, I. Sidorenko, C. H. Jaroschek et al., Phys. Plasmas 10, 3521 (2003).

${ }^{13}$ I. Shinohara and M. Fujimoto, Phys. Rev. Lett. (submitted).

${ }^{14}$ P. Ricci, J. U. Brackbill, W. Daughton, and G. Lapenta, Phys. Plasmas 11, 4489 (2004).

${ }^{15}$ W. Daughton, G. Lapenta, and P. Ricci, Phys. Rev. Lett. 93, 105004 (2004).

${ }^{16}$ G. Lapenta, J. U. Brackbill, and W. Daughton, Phys. Plasmas 10, 1577 (2003).

${ }^{17}$ W. Daughton, Phys. Plasmas 9, 3668 (2002).

${ }^{18}$ G. Lapenta and J. U. Brackbill, Phys. Plasmas 9, 1594 (2002).

${ }^{19}$ H. Karimabadi, W. Daughton, and K. B. Quest, Geophys. Res. Lett. 31, L18801 (2004).

${ }^{20}$ J. Birn, J. F. Drake, M. A. Shay et al., J. Geophys. Res. 106, 3715 (2001).

${ }^{21}$ E. G. Harris, Nuovo Cimento 23, 115 (1962).

${ }^{22}$ W. Daughton, Phys. Plasmas 6, 1329 (1999).

${ }^{23}$ R. Morse and C. Nielson, Phys. Fluids 14, 830 (1971).

${ }^{24}$ D. Forslund, Space Sci. Rev. 42, 3 (1985).

${ }^{25}$ J. U. Brackbill and D. W. Forslund, in Multiple Times Scales, edited by J. U. Brackbill and B. I. Cohen (Academic, Orlando, 1985), p. 271.

${ }^{26}$ H. X. Vu and J. U. Brackbill, Comput. Phys. Commun. 69, 253 (1992).

${ }^{27}$ P. Ricci, G. Lapenta, and J. U. Brackbill, J. Comput. Phys. 183, 117 (2002).

${ }^{28}$ R. Horiuchi and T. Sato, Phys. Plasmas 6, 4565 (1999).

${ }^{29}$ J. F. Drake, N. T. Gladd, and J. D. Huba, Phys. Fluids 24, 78 (1981).

${ }^{30}$ P. Ricci, G. Lapenta, and J. U. Brackbill, Geophys. Res. Lett. 31, L06901 (2004).

${ }^{31}$ R. Nakamura, W. Baumjohann, A. Runov et al., "Thin current sheets measured by Cluster at $250 \mathrm{Km}$ tetrahedron scale," The second workshop 
on thin current sheets, College Park, MD, 19-21 April 2004 (http:// www.glue.umd.edu/sitnov/TCS/tcs_1_files/ONLINE/

TCS_Cluster_2_RN.pdf).

${ }^{32}$ V. A. Sergeev, A. Runov, W. Baumjohann et al., Geophys. Res. Lett. 30, 1327 (2003).

${ }^{33}$ A. Runov, R. Nakamura, W. Baumjohann et al., Geophys. Res. Lett. 30, 1036 (2003).

${ }^{34}$ Y. Ren et al., Bull. Am. Phys. Soc. 48(7), 154 (2003).

${ }^{35}$ M. I. Sitnov, M. Swisdack, J. F. Drake, and P. N. Guzdar, Geophys. Res. Lett. 31, L09805 (2004).

${ }^{36}$ H. Karimabadi, P. L. Pritchett, W. Daughton et al., J. Geophys. Res. 108, 1401 (2003).

${ }^{37}$ M. A. Shay, J. F. Drake, R. E. Denton, and D. Biskamp, J. Geophys. Res. 103, 9165 (1998).

${ }^{38}$ M. Swisdack, J. F. Drake, J. G. McIlhargey, and M. A. Shay, "The transition from anti-parallel to component magnetic reconnection," J. Geophys. Res. (submitted).
${ }^{39}$ D. Biskamp, R. Z. Sagdeev, and K. Shindler, Cosm. Electrodyn. 1, 297 (1970).

${ }^{40}$ J. F. Drake and Y. C. Lee, Phys. Rev. Lett. 39, 453 (1977).

${ }^{41}$ M. M. Kuznetsova and L. M. Zelenyi, Plasma Phys. Controlled Fusion 32, 1183 (1990).

${ }^{42}$ B. Coppi, Astrophys. J., Lett. Ed. 273, L101 (1983).

${ }^{43}$ J. Chen and P. Palmadesso, Phys. Fluids 27, 1198 (1984).

${ }^{44}$ J. Chen and Y. C. Lee, Phys. Fluids 28, 2137 (1985).

${ }^{45}$ D. V. Forslund and B. Coppi, Bull. Am. Phys. Soc. 13, 306 (1968).

${ }^{46}$ J. Ambrosiano, L. C. Lee, and Z. F. Fu, J. Geophys. Res. 91, 113 (1986).

${ }^{47}$ Y. Shi, L. C. Lee, and Z. F. Fu, J. Geophys. Res. 92, 12171 (1987).

${ }^{48}$ E. Camporeale and G. Lapenta, "Model of bifurcated current sheets in the Earth's magnetotail: equilibrium and stability," J. Geophys. Res. (to be published).

${ }^{49}$ S. W. H. Cowley, Planet. Space Sci. 26, 1037 (1978).

${ }^{50}$ J. Birn, K. Shindler, and M. Hesse, J. Geophys. Res. 109, A02217 (2004). 